\title{
Impact of CALGB 9343 Trial and Sociodemographic Variation on Patterns of Adjuvant Radiation Therapy Practice for Elderly Women ( $\geq 70$ Years) with Stage I, Estrogen Receptor-positive Breast Cancer: Analysis of the National Cancer Data Base
}

\author{
QUYEN D. CHU ${ }^{1,2}$, MEIJIAO ZHOU ${ }^{3}$, KAELEN L. MEDEIROS $^{3}$, PRAKASH PEDDI $^{2,4}$ and XIAO CHENG WU ${ }^{3}$ \\ Departments of ${ }^{1}$ Surgery, and ${ }^{4}$ Medicine, Louisiana State University Health \\ Sciences Center in Shreveport, Shreveport, Louisiana, LA, U.S.A.; \\ ${ }^{2}$ Feist-Weiller Cancer Center, Shreveport, LA, U.S.A.; \\ ${ }^{3}$ School of Public Health, Louisiana State University Health Sciences Center, New Orleans, LA, U.S.A.
}

\begin{abstract}
Background: The Cancer and Leukemia Group B (CALGB) 9343 trial demonstrated that adjuvant radiation therapy (RT) can be omitted in women 70 years or older, with small $(\leq 2 \mathrm{~cm})$, negative lymph nodes, estrogen receptor $(E R)$ positive breast cancer. We examined whether RT usage following the CALGB publication had decreased over time and evaluated sociodemographic and clinical factors associated with RT omission. Materials and Methods: From the National Cancer Data Base, we analyzed a cohort of 120,308 women aged 70 years or older with stage I, ER-positive breast cancer who underwent lumpectomy. Patients were classified into two groups based on the time of CALGB 9343 publication: (i) pre-CALGB (up to 2004), and (ii) post-CALGB (2005-2012). Clinicopathological and sociodemographic variables were compared between pre- and post-CALGB groups. Chi-square and multivariable logistic regression were employed, with the omission of adjuvant $R T$ as the primary outcome in the regression analysis. Results: Radiation therapy usage decreased by $4.1 \%$ after $C A L G B$ publication (on average $71.6 \%$ pre$C A L G B$ vs. $67.5 \%$ post-CALGB; $p<0.0001)$. Almost one-third of
\end{abstract}

Presented at the Southern Surgical Association, 127th Annual Meeting, The Omni Homestead Resort, Hot Springs, Virginia, December 2015.

Correspondence to: Prakash Peddi, MD, MS, Assistant Professor of Medicine, Division of Hematology and Oncology, Louisiana State University Health Sciences Center-Shreveport, Feist-Weiller Cancer Center Shreveport, LA - 71130, U.S.A. Tel: +1 3188131057, Fax: +1 3188131055, e-mail: ppeddi@1suhsc.edu

Key Words: Elderly, breast cancer, hormone-positive breast cancer, $\mathrm{ER}^{+}$, stage I breast cancer, radiation therapy, small breast cancer, adherence. women aged $\geq 85$ years received $R T$ in the post-CALGB group. In a multivariable model, the variables significantly associated with increased odds for omission of $R T$ in the post-CALGB group were: advanced age, African-American, increased great circle distance, therapy under academic research program, residents of East South-Central region, living in a rural population $<2,500$ not adjacent to a metropolitan area, low income level, Medicaid recipients, high comorbidity index, small tumor, well-differentiated histology, residual tumor, and lack of receipt of chemotherapy and anti-hormonal therapy. Conclusion: During the study period, the CALGB trial publication had a minimal impact on the rate of adjuvant $R T$ use among elderly women with small, ER-positive breast cancers. Significant variation in RT usage existed across sociodemographic strata.

In 2004, Cancer and Leukemia Group B (CALGB) 9343 published a compelling phase III trial that demonstrated no survival difference among elderly women (aged $\geq 70$ years) with small $(\leq 2 \mathrm{~cm})$ estrogen receptor-positive $\left(\mathrm{ER}^{+}\right)$tumors treated by breast-conserving therapy (BCT) and antihormonal therapy who were randomized to receive or not receive adjuvant radiation therapy (RT) (1). The authors posited that adjuvant RT can safely be omitted in this highly selected group of patients with breast cancer. Subsequent to CALGB publication, the National Comprehensive Cancer Network (NCCN) released its national practice guidelines supporting CALGB's position in 2004. However, after these reports, a number of investigators found that the results of CALGB were not rapidly incorporated into the general practice of the community. There was minimal impact on practice patterns following the CALGB publication: absolute reduction of RT usage following publication was only $4 \%$ to 9\% over a period of 3-9 years after publication (2-4). Unfortunately, these reports were either based on large 
administrative databases linked to Surveillance, Epidemiology, and End Results (SEER) (2,3), highly selective institutions (4) that may not represent the general population, or lacked detailed clinical, pathological and sociodemographic information. Although SEER data are population-based, they only cover certain US areas. Our study conducted an analysis to identify factors associated with RT omission using the National Cancer Data Base (NCDB), and to our knowledge, this is the largest cohort of patients (over $113,000)$ who fit CALGB criteria for which such an analysis has been performed. The purpose of this study was to examine changes in use of RT pre- and post-CALGB publication and identify clinical and sociodemographic factors associated with the omission of adjuvant RT.

\section{Materials and Methods}

Data source. The NCDB is a joint project of the Commission on Cancer $(\mathrm{CoC})$ of the American College of Surgeons and the American Cancer Society. More than 1,500 CoC-accredited facilities contribute clinical information to the database. Approximately $70 \%$ of newly diagnosed cancer cases in the US and 30 million historical records are captured in the database. Access to NCDB was awarded to the investigators after having successfully applied for the NCDB Participant User File (PUF). The data sets in the PUF were deidentified and were in compliance with the privacy requirements of the Health Insurance Portability and Accountability Act. The study was exempted from Institutional Review Board approval by the Louisiana State University Health Sciences Center-Shreveport.

Study population. A total of $120,308 \mathrm{ER}^{+}$breast cancer cases from 2004 to 2012 among women aged 70 years or more, with tumor size $\leq 2 \mathrm{~cm}$ and lymph node-negative disease, who underwent BCT were analyzed to identify factors significantly associated with omission of adjuvant RT. The CALGB trial, which included $\mathrm{ER}^{+}$patients, lacks data on progesterone receptor (PR) status. We have included all $\mathrm{ER}^{+}$patients, regardless of PR status. Patients were classified into two groups based on the time of CALGB 9343 publication: (i) pre-CALGB (up to 2004), and (ii) post-CALGB (2005-2012).

According to the NCDB's PUF dictionary, facility type was classified as (i) community cancer program, (ii) comprehensive community cancer program, (iii) academic research program, and (4) others (5). Community cancer programs are those that treat between 100 and 500 newly diagnosed patients with cancer each year, and patients may be referred to another facility for part of their diagnosis or treatment (5). Comprehensive community cancer programs are those that treat more than 500 newly-diagnosed patients each year (5). Academic and research institutions are those that treat more than 500 newly diagnosed patients each year while offering postgraduate medical education programs (5).

Facility location was categorized into regions within the United States based on the US. Census Regions and Divisions: (i) New England, (ii) Mid-Atlantic, (iii) South Atlantic, (iv) East North Central, (v) East South Central, (vi) West North Central, (vii) West South Central, (viii) Mountain, and (ix) Pacific.

Race/ethnicity was categorized as White, African-American, American Indian/Aleutian or Eskimo, Asian or Pacific Islanders, and Others. Insurance status was classified as (i) uninsured, ii) private insurance/managed care, (iii) Medicaid, (iv) Medicare, and (v) other governmental insurance. Patients with both private insurance and Medicare were grouped in the private insurance category. Median household income level was classified as (i) $<\$ 38,000$, (ii) $\$ 38,000$ $\$ 47,999$, (iii) $\$ 48,000-\$ 62,999$, (4) $\geq \$ 63,000$. Education level was classified into percentage of adults (age $\geq 25$ years) who did not graduate from high school in the area based on the 2012 American Community Survey data: (i) $\geq 21 \%$, (ii) $13-20.9 \%$, (iii) $7-12.9 \%$, (iv) $<7 \%$. Comorbidity/Deyo score was reported as 0,1 or $2(6,7)$.

Population density was classified as (i) metropolitan with $\geq 1$ million residents, (ii) metropolitan with 250,000 to 1 million, (iii) urban with $<250,000$, (iv) urban with $\geq 20,000$ and adjacent to a metropolitan area, (v) urban with $\geq 20,000$ but not adjacent to a metropolitan area, (vi) urban with $<20,000$ and adjacent to a metropolitan area, (vii) urban with $<20,000$ but not adjacent to a metropolitan area, (viii) rural with $<2,500$ and adjacent to a metropolitan area, and (ix) rural with $<2,500$ but not adjacent to a metropolitan area. Great circle distance is measured in miles and defined as being that between the patient's residence at diagnosis and the hospital that reported the case.

Statistical analysis. The nonparsimonious approach using age, ethnicity, etc. was used to construct models. The percentage of patients who did not receive RT was calculated across strata of covariates for the pre- and post-CALGB groups, and Chi-square tests were used to compare the pre- and the post-CALGB group results. The absolute percentage change between the samples of each stratum of covariates was also calculated.

Multivariable logistic regression analysis was applied to adjust for the covariates when determining the independent significant factors associated with omission of RT usage, and the results are reported using odds ratio (OR) and 95\% confidence interval (CI). Differences with a $p$-value of 0.05 or less was considered statistically significant. All statistical analyses were performed using SAS Version 9.4 statistical software, (SAS Institute Inc., Cary, NC, USA).

\section{Results}

Postoperative RT was omitted in $32.1 \%$ of the 120,308 women with breast cancer (Table I). Table I shows results from the Chi-Square test comparing the distribution of variables associated with omission of RT for the two-time periods. Approximately $28.4 \%$ of patients in the pre-CALGB group omitted RT versus $32.5 \%$ in the post-CALGB group $(p<0.01)$. This translates to an absolute decrease of $4.04 \%$ in RT use following the initial CALGB 9343 report (Table I and Figure 1). Note that the trend line after year 2004 showed very little variation in the use of RT (Figure 1). Among the different age groups, the greatest decline in RT utilization was observed among the octogenarians (ages 8084 years). Despite such a decline, $58.4 \%$ of patients aged 80 84 years and $33 \%$ of patients aged $\geq 85$ years continued to receive RT in the post-CALGB group.

Whites, African-Americans, and Asian/Pacific Islander experienced significant reduction of RT usage. The absolute RT reduction for Whites, African-Americans, and Asian/Pacific Islander was $3.86 \%(p<0.0001), 6.80 \%(p=0.001)$, and $10.42 \%$, 
respectively $(p<0.01)$. Although the majority of cancer programs witnessed a significant decrease in RT usage, the greatest decline was seen in those in a community cancer program $(5.25 \%$; $p<0.01)$ followed by academic research program $(4.59 \%$; $p<0.01)$ and comprehensive community cancer program $(3.58 \%$; $p<0.01)$. When examining RT usage among the different regions of the US, there was wide variation; Pacific followed by New England regions had the highest decrease in RT usage while Mid-Atlantic, West North Central, and West South Central had no significant changes in RT usage (Figure 2).

Using multivariable logistic regression analyses, the following variables were found to be independent predictors of RT omission following CALGB publication: age, ethnicity, facility type, facility location, population density, great circle distance, primary payor, income, comorbidity index, tumor size, grade, status of surgical margins, and lack of receipt of chemotherapy and anti-hormonal therapy (Table II). When analyzed in greater detail, the following were associated with a higher likelihood of RT omission: advanced age $(\geq 85$ years), African-American race, academic research programs, East South Central, South Atlantic, and New England regions, Medicaid recipients, low income level, high comorbidity index, small tumors, positive margins, and lack of chemotherapy and anti-hormonal therapy.

Octogenarians $\geq 85$ years were more likely not to receive RT than those aged 70-74 years $(\mathrm{OR}=7.90,95 \% \mathrm{CI}=7.51$ 8.30). Compared to White patients, African-American patients were more likely not to receive $\mathrm{RT}(\mathrm{OR}=1.11,95 \% \mathrm{CI}=1.04$ 1.19). Academic research programs were more likely to omit RT compared to community cancer programs $(\mathrm{OR}=1.14,95 \%$ $\mathrm{CI}=1.08-1.21)$. Compared to Pacific region, East SouthCentral region $(\mathrm{OR}=1.32,95 \% \mathrm{CI}=1.21-1.43)$, South Atlantic region $(\mathrm{OR}=1.18,95 \% \mathrm{CI}=1.11-1.24)$, and New England region $(\mathrm{OR}=1.12,95 \% \mathrm{CI}=1.04-1.20)$ were more likely to omit RT. Patients residing $\geq 50$ miles from the hospital that reported the case were more likely not to receive RT than those residing $<50$ miles $(\mathrm{OR}=1.36,95 \% \mathrm{CI}=1.26-1.47)$.

Compared to those with private insurance, those with Medicaid were more likely to omit RT (OR=1.25, 95\% $\mathrm{CI}=1.06-1.49)$. Those in the lowest income bracket $(\$ 38,000)$ were more likely not to receive RT compared to those in the highest income bracket $(\$ 63,000)(\mathrm{OR}=1.10,95 \% \mathrm{CI}=1.03$ 1.17). Patients with comorbidity/Deyo score of $\geq 2$ were more likely to omit $\mathrm{RT}(\mathrm{OR}=1.89,95 \% \mathrm{CI}=1.75-2.05)$. Compared to patients whose tumors measured $>1.0-2.0 \mathrm{~cm}$, those measuring $>0.1-0.5 \mathrm{~cm}$ were more likely to omit RT $(\mathrm{OR}=1.22, \mathrm{CI}=1.16-1.28)$. Those with residual tumor were more likely not to receive RT compared to those without residual tumor $(\mathrm{OR}=1.25,95 \% \mathrm{CI}=1.16-1.34)$. Patients who did not receive chemotherapy $(\mathrm{OR}=1.34,95 \% \mathrm{CI}=1.21-1.48)$ and those who did not receive anti-hormonal therapy were also more likely not to receive RT compared to those who $\operatorname{did}(\mathrm{OR}=2.28,95 \% \mathrm{CI}=2.21-2.36)$.
Note that socioeconomic status such as education level had no significant impact on RT utilization. Table III summarizes the patterns of RT utilization. Note that in the pre-CALGB era, $82.9 \%$ had external beam radiation; in the post-CALGB era, $65.1 \%$ had external beam radiation, while $13.8 \%$ had brachytherapy. Table IV summarizes the findings of studies that evaluated the impact of CALGB 9343 on practice patterns across the US.

\section{Discussion}

The National Surgical Adjuvant Breast and Bowel Project (NSABP) B-06 (8) and the latest publication from the Early Breast Cancer Trialists' Collaborative Group (EBCTCG) (9) established that adjuvant RT after BCT improves outcome for patients with operable breast cancer. In NSABP B-06, the cumulative incidence of ipsilateral breast cancer recurrence 20 years after surgery was $14.3 \%$ for women who had adjuvant RT after a lumpectomy versus $39.2 \%$ for those with lumpectomy alone $(p<0.01)(10)$. However, NSABP B-06 inclusion criteria were broad and included women with tumor measuring up to $4 \mathrm{~cm}$ and those with positive lymph nodes. The EBCTCG reported that adjuvant RT reduces recurrence and death compared to the non-RT group. However, the absolute 10-year recurrence reduction was not uniformly observed across the age strata; younger age groups ( $24.6 \%$ for those aged $<40$ years) had a more substantial reduction in recurrence than the older age groups $(17.7 \%$ for those aged $\geq 70$ years) (9). Given the small benefit of RT for older women, many began to question its utility among elderly women who were considered to harbor low-risk breast cancer

The CALGB 9343 trial was seminal in that evaluated the efficacy of radiotherapy after breast-conserving surgery in older women with low risk breast cancer. It recruited over 600 women ages $\geq 70$ years with estrogen-receptor positive stage I breast cancer (T1N0M0), who were treated with breast-conserving therapy and adjuvant tamoxifen (1). The patients were then randomized to either the adjuvant RT group or the non-RT group. The initial results published in 2004 found that at a median follow-up of five years, the incidence of ipsilateral breast recurrence (IBR) or regional nodal recurrence was $4 \%$ in the non-RT group versus $1 \%$ for the RT group $(p<0.01)$. However, there were no significant differences in breast-cancer specific survival, overall survival (OS), time to mastectomy, time to distant metastases, and cancer-specific survival between the two groups, although the RT arm had significant adverse events such as breast edema, pain and fibrosis, and retraction (1). These results led the National Comprehensive Cancer Network (NCCN) to incorporate into their guidelines that omission of RT in elderly women who met CALGB criteria should be given serious consideration (11). 
Table I. Factors associated with omission of adjuvant radiation therapy (Chi-square test).

\begin{tabular}{|c|c|c|c|c|c|c|}
\hline \multirow[b]{2}{*}{ Variable } & \multicolumn{6}{|c|}{ Not receiving RT, \% } \\
\hline & $\begin{array}{c}\text { Total group } \\
(\mathrm{N}=120,308)\end{array}$ & Overall & $\begin{array}{c}\text { Pre-CALGB } \\
(\mathrm{N}=11,172)\end{array}$ & $\begin{array}{c}\text { Post CALGB } \\
(\mathrm{N}=109,136)\end{array}$ & $\begin{array}{l}\text { Absolute change } \\
\text { (pre-post)* }\end{array}$ & $p$-Value \\
\hline Total patients & & & 9.3 & 90.7 & & \\
\hline Overall & 100 & 32.1 & 28.4 & 32.5 & -4.04 & $<0.0001$ \\
\hline \multicolumn{7}{|l|}{ Age (years) } \\
\hline $70-74$ & 36.0 & 19.4 & 17.6 & 19.6 & -1.92 & 0.004 \\
\hline $75-79$ & 30.4 & 26.9 & 23.6 & 27.2 & -3.60 & $<0.0001$ \\
\hline $80-84$ & 21.2 & 40.9 & 34.8 & 41.6 & -6.84 & $<0.0001$ \\
\hline $85+$ & 12.4 & 66.6 & 62.4 & 67.0 & -4.57 & 0.001 \\
\hline \multicolumn{7}{|l|}{ Race/ethnicity } \\
\hline White & 91.4 & 32.0 & 28.5 & 32.4 & -3.86 & $<0.0001$ \\
\hline African American & 5.8 & 34.1 & 27.9 & 34.7 & -6.80 & 0.0009 \\
\hline American Indian, Aleutian, or Eskimo & 0.1 & 28.8 & 14.3 & 29.7 & -15.37 & 0.67 \\
\hline Asian/Pacific Islander & 1.5 & 26.4 & 16.8 & 27.2 & -10.42 & 0.0067 \\
\hline Missing, not available, or unknown & 1.2 & 35.5 & 36.3 & 35.4 & 0.94 & 0.83 \\
\hline \multicolumn{7}{|l|}{ Facility type } \\
\hline Community cancer program & 12.4 & 33.0 & 28.2 & 33.5 & -5.25 & $<0.0001$ \\
\hline Comprehensive community cancer center & 62.0 & 30.8 & 27.5 & 31.1 & -3.58 & $<0.0001$ \\
\hline Academic research program & 25.5 & 34.9 & 30.7 & 35.3 & -4.59 & $<0.0001$ \\
\hline Other specified type of program & 0.2 & 35.0 & 33.3 & 35.2 & -1.86 & 0.89 \\
\hline \multicolumn{7}{|l|}{ Facility location } \\
\hline New England & 8.2 & 35.0 & 29.4 & 35.6 & -6.20 & $<0.0001$ \\
\hline Mid Atlantic & 16.6 & 31.3 & 29.5 & 31.5 & -1.99 & 0.07 \\
\hline South Atlantic & 21.5 & 34.9 & 31.6 & 35.2 & -3.63 & 0.0004 \\
\hline East North Central & 19.3 & 28.9 & 25.2 & 29.2 & -4.02 & $<0.0001$ \\
\hline East South Central & 4.5 & 35.8 & 31.1 & 36.2 & -5.11 & 0.0351 \\
\hline West North Central & 7.3 & 28.6 & 25.9 & 28.8 & -2.95 & 0.09 \\
\hline West South Central & 5.4 & 32.7 & 30.6 & 32.9 & -2.27 & 0.28 \\
\hline Mountain & 4.4 & 32.5 & 26.8 & 33.0 & -6.18 & 0.0092 \\
\hline Pacific & 12.8 & 31.8 & 26.1 & 32.4 & -6.30 & $<0.0001$ \\
\hline \multicolumn{7}{|l|}{ Urban/rural location } \\
\hline $\mathrm{MA} \geq 1$ million & 52.2 & 32.7 & 30.2 & 32.9 & -2.73 & $<0.0001$ \\
\hline MA 250,000 to 1 million & 21.9 & 33.0 & 26.6 & 33.7 & -7.01 & $<0.0001$ \\
\hline Urban $<250,000$ & 9.9 & 28.4 & 25.2 & 28.7 & -3.56 & 0.0128 \\
\hline Urban $\geq 20,000$ adjacent to MA & 3.9 & 29.0 & 22.3 & 29.7 & -7.31 & 0.0025 \\
\hline Urban $\geq 20,000$ not adjacent to MA & 1.3 & 27.3 & 21.8 & 27.8 & -5.98 & 0.14 \\
\hline Urban $<20,000$ adjacent to MA & 4.1 & 29.9 & 27.4 & 30.1 & -2.65 & 0.24 \\
\hline Urban $<20,000$ not adjacent to MA & 1.9 & 29.4 & 24.5 & 29.9 & -5.38 & 0.11 \\
\hline Rural $<2500$ adjacent to MA & 0.6 & 32.7 & 31.7 & 32.8 & -1.15 & 0.84 \\
\hline Rural $<2500$ not adjacent to MA & 0.7 & 33.1 & 30.7 & 33.3 & -2.68 & 0.67 \\
\hline Missing information & 3.5 & 37.1 & 31.6 & 37.8 & -6.27 & 0.0081 \\
\hline \multicolumn{7}{|l|}{ Great circle distance } \\
\hline$<50$ miles & 93.9 & 31.8 & 28.1 & 32.2 & -4.11 & $<0.0001$ \\
\hline$\geq 50$ miles & 4.8 & 35.7 & 33.4 & 35.9 & -2.47 & 0.28 \\
\hline Missing information & 1.4 & 37.3 & 32.4 & 38.2 & -5.77 & 0.08 \\
\hline \multicolumn{7}{|l|}{ Primary Payor } \\
\hline Uninsured & 0.3 & 37.4 & 34.5 & 37.7 & -3.19 & 0.74 \\
\hline Private insurance & 11.6 & 29.2 & 26.8 & 29.4 & -2.61 & 0.0477 \\
\hline Medicaid & 0.8 & 35.1 & 28.0 & 35.5 & -7.46 & 0.28 \\
\hline Medicare & 85.8 & 32.4 & 28.6 & 32.8 & -4.24 & $<0.0001$ \\
\hline Other government & 0.3 & 33.5 & 33.3 & 33.5 & -0.20 & 0.99 \\
\hline Missing, not available, or unknown & 1.2 & 33.1 & 30.2 & 33.6 & -3.36 & 0.34 \\
\hline \multicolumn{7}{|l|}{ Income } \\
\hline$<\$ 38,000$ & 13.1 & 33.9 & 29.5 & 34.4 & -4.91 & 0.0001 \\
\hline$\$ 38,000-47,999$ & 22.1 & 32.2 & 27.2 & 32.7 & -5.42 & $<0.0001$ \\
\hline$\$ 48,00-62,999$ & 27.4 & 31.8 & 28.0 & 32.1 & -4.10 & $<0.0001$ \\
\hline$\$ 63,000+$ & 35.9 & 31.4 & 28.8 & 31.7 & -2.87 & 0.0002 \\
\hline
\end{tabular}


Table I. Continued

\begin{tabular}{|c|c|c|c|c|c|c|}
\hline \multirow[b]{2}{*}{ Variable } & \multicolumn{6}{|c|}{ Not receiving RT, \% } \\
\hline & $\begin{array}{l}\text { Total group } \\
(\mathrm{N}=120,308)\end{array}$ & Overall & $\begin{array}{c}\text { Pre-CALGB } \\
(\mathrm{N}=11,172)\end{array}$ & $\begin{array}{l}\text { Post CALGB } \\
(\mathrm{N}=109,136)\end{array}$ & $\begin{array}{l}\text { Absolute change } \\
\text { (pre-post)* }\end{array}$ & $p$-Value \\
\hline Missing information & 1.5 & 37.2 & 31.9 & 38.2 & -6.22 & 0.0543 \\
\hline \multicolumn{7}{|l|}{ Education** } \\
\hline$\geq 21 \%$ & 11.4 & 34.3 & 30.8 & 34.7 & -3.90 & 0.0054 \\
\hline $13-20.9 \%$ & 22.4 & 32.4 & 28.2 & 32.8 & -4.56 & $<0.0001$ \\
\hline $7-12.9 \%$ & 35.4 & 31.4 & 27.8 & 31.8 & -3.98 & $<0.0001$ \\
\hline$<7 \%$ & 29.4 & 31.6 & 28.1 & 31.9 & -3.79 & $<0.0001$ \\
\hline Missing information & 1.4 & 37.3 & 31.8 & 38.3 & -6.46 & 0.0470 \\
\hline \multicolumn{7}{|l|}{ Comorbidities/Deyo score } \\
\hline 0 & 81.9 & 30.9 & 27.5 & 31.2 & -3.76 & $<0.0001$ \\
\hline 1 & 14.8 & 36.1 & 33.9 & 36.3 & -2.42 & 0.08 \\
\hline 2 & 3.3 & 44.4 & 35.2 & 45.0 & -9.84 & 0.0023 \\
\hline \multicolumn{7}{|l|}{ Tumor size } \\
\hline$\leq 0.1 \mathrm{~cm}$ & 1.8 & 33.6 & 31.5 & 33.9 & -2.39 & 0.48 \\
\hline$>0.1-0.5 \mathrm{~cm}$ & 12.4 & 35.3 & 29.2 & 35.9 & -6.69 & $<0.0001$ \\
\hline$>0.5-1.0 \mathrm{~cm}$ & 36.5 & 32.7 & 28.4 & 33.1 & -4.70 & $<0.0001$ \\
\hline$>1.0-2.0 \mathrm{~cm}$ & 47.1 & 30.6 & 27.9 & 30.9 & -3.06 & $<0.0001$ \\
\hline Missing information & 2.2 & 34.3 & 32.2 & 34.7 & -2.49 & 0.35 \\
\hline \multicolumn{7}{|l|}{ Grade } \\
\hline Well differentiated & 38.5 & 34.2 & 29.8 & 34.6 & -4.89 & $<0.0001$ \\
\hline Moderately differentiated & 45.6 & 31.1 & 27.9 & 31.4 & -3.51 & $<0.0001$ \\
\hline Poorly differentiated & 10.2 & 28.3 & 26.2 & 28.5 & -2.33 & 0.09 \\
\hline Undifferentiated, anaplastic & 0.1 & 26.3 & 28.6 & 25.8 & 2.79 & 0.76 \\
\hline Cell type not determined, unavailable & 5.5 & 33.0 & 28.2 & 33.7 & -5.46 & 0.0015 \\
\hline \multicolumn{7}{|l|}{ Surgical margins } \\
\hline No residual tumor & 94.8 & 31.6 & 27.6 & 32.0 & -4.43 & $<0.0001$ \\
\hline With residual tumor & 4.4 & 41.4 & 42.0 & 41.3 & 0.77 & 0.72 \\
\hline Missing information & 0.8 & 40.2 & 37.4 & 40.6 & -3.23 & 0.51 \\
\hline \multicolumn{7}{|l|}{ Chemotherapy } \\
\hline None & 94.5 & 32.6 & 28.6 & 33.0 & -4.36 & $<0.0001$ \\
\hline Yes & 3.3 & 18.2 & 16.5 & 18.3 & -1.86 & 0.46 \\
\hline Missing information & 2.2 & 32.4 & 31.0 & 32.6 & -1.58 & 0.57 \\
\hline \multicolumn{7}{|l|}{ Anti-hormonal therapy } \\
\hline None & 32.1 & 47.7 & 42.4 & 48.4 & -5.99 & $<0.0001$ \\
\hline Yes & 64.1 & 23.9 & 18.8 & 24.4 & -5.60 & $<0.0001$ \\
\hline Missing information & 3.8 & 38.3 & 30.0 & 39.4 & -9.33 & $<0.0001$ \\
\hline
\end{tabular}

CALGB: Cancer and Leukemia Group B; pre-CALGB: up to 2004; post-CALGB: 2005-2012; MA: metropolitan area; RT: radiation.*Percentages were rounded to nearest 10th. **Percentage of adults (age $\geq 25$ years) who did not graduate from high school in the area based on the 2012 American Community Survey data.

A long-term update of CALGB 9343 confirmed the durability of these results (12). With a median follow-up of 12.6 years, the locoregional recurrence rate was $2 \%$ in the RT group versus $10 \%$ in the non-RT group $(p<0.001)$; the 10 -year OS was not significantly different between the RT group (67\%) and the non-RT group (66\%). Although the locoregional recurrence rate was significantly different, the authors indicated that the difference was not clinically significant and concluded that RT can be safely omitted in this select group of patients (12).
A recent publication from the United Kingdom (PRIME II trial) also confirmed the permissibility of omitting adjuvant RT in older women with small breast cancer who underwent BCT (13). More than 1,300 women ages $\geq 65$ years with $\leq 3$ $\mathrm{cm}$ tumor, grade 3 histology or lymphovascular invasion, but not both, and clear margins were randomized into the RT versus the non-RT groups. All women received adjuvant endocrine therapy. After a median follow-up of 5 years, IBR was $1.3 \%$ in the RT group versus $4.1 \%$ in the non-RT group $(p=0.0002)$. Although local relapses were statistically 


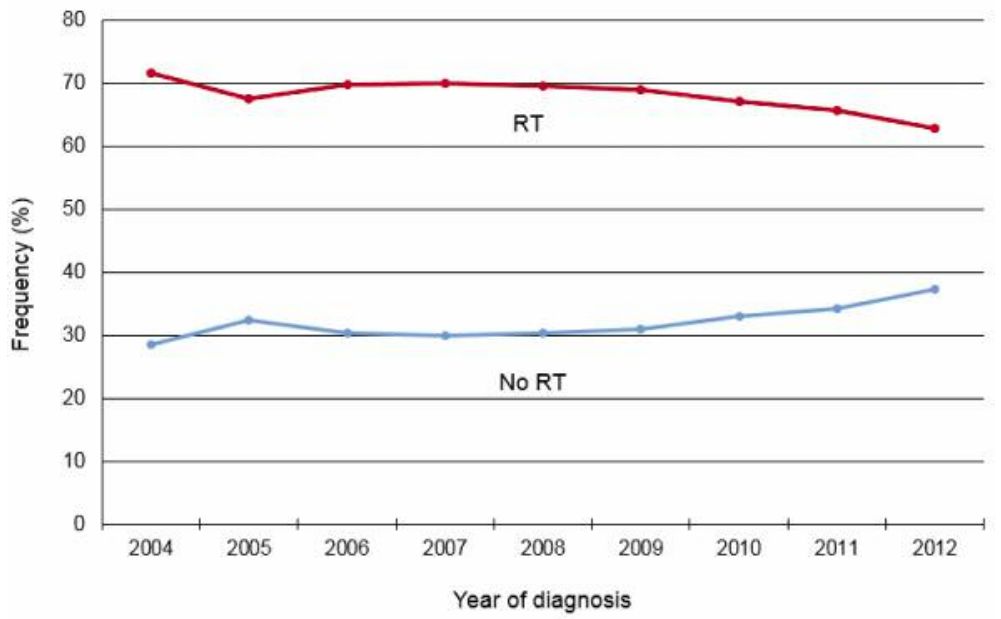

Figure 1. Radiation $(R T)$ trends over the decades for elderly patients ( $\geq 70$ years) with small, estrogen receptor-positive breast cancer treated with breast-conserving therapy: note that RT utilization remained relatively constant over the decades, despite 2004 publication of Cancer and Leukemia Group B 9343 trial results.

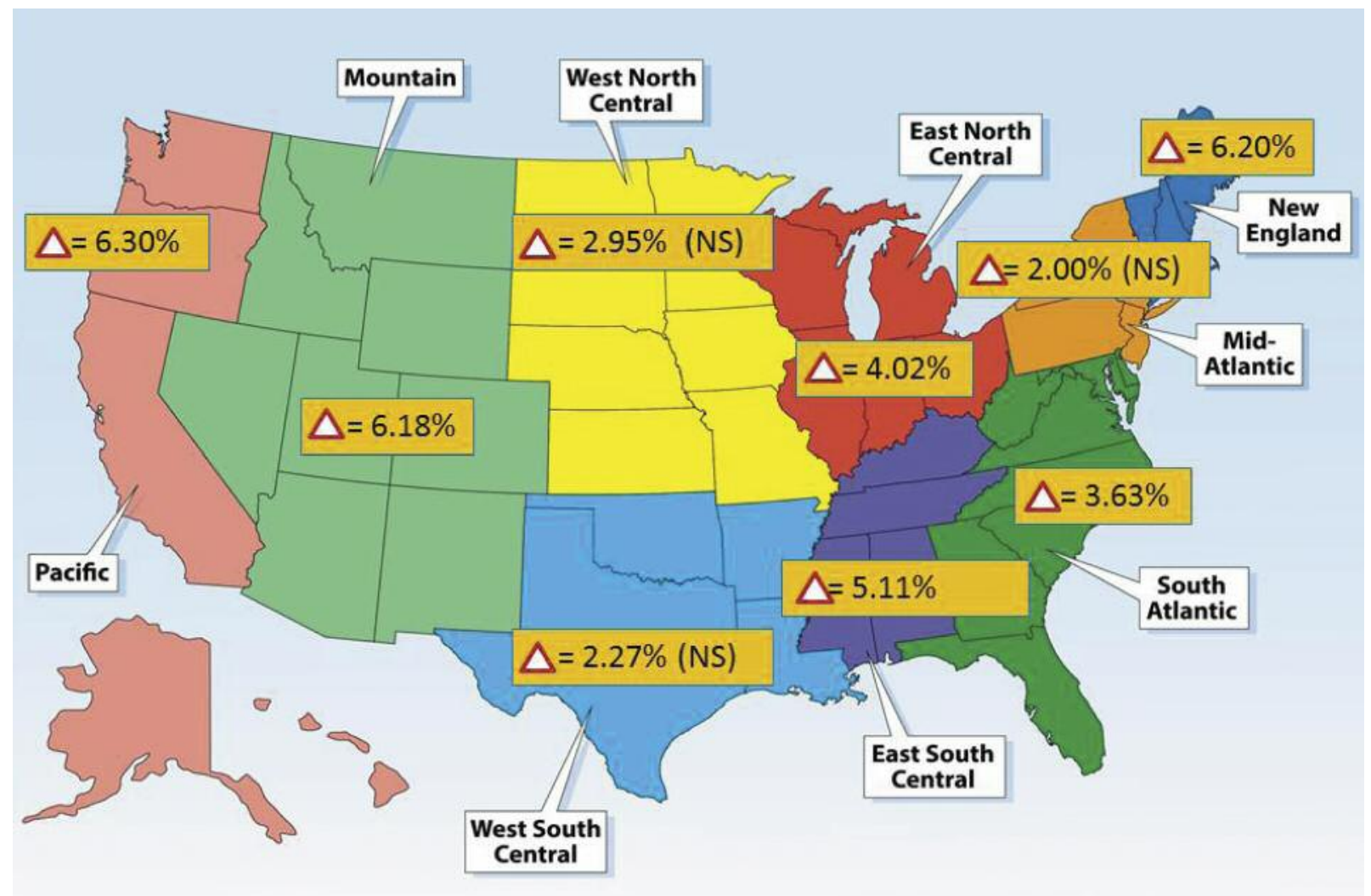

Figure 2. Absolute reduction (4) in administration of radiation therapy (\%) over the two calendar periods (up to 2004 and 2005-2012). There was a wide variation of radiation utilization over the nine different regions of the US following publication of Cancer and Leukemia Group B 9343 trial results. Note that patients in most regions experienced significant reduction of radiation utilization, except for West North Central region, West South Central, East South Central and Mid-Atlantic, which did not. 
Table II. Results from multivariable logistic regression model used to Identify factors associated with radiotherapy omission post-Cancer and Leukemia Group B Publication (2005-2012) (N=90,278). The selected variables were based on results from the literature and our chi-square test; complete case analysis was used to handle the missing data.

\begin{tabular}{|c|c|c|c|c|c|c|c|}
\hline Variable & $\begin{array}{l}\text { Odds } \\
\text { ratio }\end{array}$ & $\begin{array}{c}95 \% \\
\text { Confidence } \\
\text { interval }\end{array}$ & $p$-Value & Variable & $\begin{array}{l}\text { Odds } \\
\text { ratio }\end{array}$ & $\begin{array}{c}95 \% \\
\text { Confidence } \\
\text { interval }\end{array}$ & $p$-Value \\
\hline Age (years) & & & & Income & & & \\
\hline 70-74 (Reference) & 1.00 & & & $\$ 63,000+($ Reference $)$ & 1.00 & & \\
\hline $75-79$ & 1.54 & $1.48-1.61$ & $<0.0001$ & $\$ 48,000-62,999$ & 1.08 & $1.03-1.13$ & 0.001 \\
\hline $80-84$ & 2.85 & $2.74-2.98$ & $<0.0001$ & $\$ 38,000-47,999$ & 1.09 & $1.04-1.15$ & 0.0012 \\
\hline $85+$ & 7.90 & $7.51-8.30$ & $<0.0001$ & $<\$ 38,000$ & 1.10 & $1.03-1.17$ & 0.0061 \\
\hline Race/ethnicity & & & & Education* & & & \\
\hline White (Reference) & 1.00 & & & $<7 \%$ (Reference) & 1.00 & & \\
\hline African American & 1.11 & $1.04-1.19$ & 0.0018 & $7-12.9 \%$ & 0.97 & $0.93-1.01$ & 0.20 \\
\hline American Indian, Aleutian, or Eskimo & 0.85 & $0.52-1.40$ & 0.53 & $13-20.9 \%$ & 0.99 & $0.94-1.05$ & 0.75 \\
\hline Asian/Pacific Islander & 0.87 & $0.76-0.99$ & 0.0344 & $\geq 21 \%$ & 1.04 & $0.97-1.11$ & 0.28 \\
\hline Facility type & & & & Comorbidities/Deyo score & & & \\
\hline Community cancer program (Reference) & 1.00 & & & 0 (Reference) & 1.00 & & \\
\hline Comprehensive community cancer center & 0.85 & $0.81-0.89$ & $<0.0001$ & 1 & 1.31 & $1.25-1.36$ & $<0.0001$ \\
\hline Academic research program & 1.14 & $1.08-1.21$ & $<0.0001$ & $\geq 2$ & 1.89 & $1.75-2.05$ & $<0.0001$ \\
\hline Facility location & & & & Tumor size & & & \\
\hline Pacific (Reference) & 1.00 & & & $>1.0-2.0 \mathrm{~cm}$ (Reference) & 1.00 & & \\
\hline New England & 1.12 & $1.04-1.20$ & 0.0022 & $>0.5-1.0 \mathrm{~cm}$ & 1.08 & $1.05-1.12$ & $<0.0001$ \\
\hline Mid Atlantic & 0.94 & $0.89-1.00$ & 0.0501 & $\leq 0.1 \mathrm{~cm}$ & 1.18 & $1.04-1.34$ & 0.0125 \\
\hline South Atlantic & 1.18 & $1.11-1.24$ & $<0.0001$ & $>0.1-0.5 \mathrm{~cm}$ & 1.22 & $1.16-1.28$ & $<0.0001$ \\
\hline East North Central & 0.85 & $0.81-0.91$ & $<0.0001$ & Grade & & & \\
\hline East South Central & 1.32 & $1.21-1.43$ & $<0.0001$ & Well differentiated (Reference) & 1.00 & & \\
\hline West North Central & 0.84 & $0.78-0.90$ & $<0.0001$ & Moderately differentiated & 0.87 & $0.84-0.90$ & $<0.0001$ \\
\hline West South Central & 1.03 & $0.95-1.11$ & 0.51 & Poorly differentiated & 0.77 & $0.72-0.81$ & $<0.0001$ \\
\hline Mountain & 1.06 & $0.98-1.16$ & 0.15 & Undifferentiated, anaplastic & 0.65 & $0.41-1.02$ & 0.06 \\
\hline Urban/rural location & & & & Surgical margins & & & \\
\hline MA $\geq 1$ million (Reference) & 1.00 & & & No residual tumor (Reference) & 1.00 & & \\
\hline MA 250,000 to 1 million & 1.01 & $0.97-1.05$ & 0.54 & With residual tumor & 1.25 & $1.16-1.34$ & $<0.0001$ \\
\hline Urban $<250,000$ & 0.85 & $0.80-0.90$ & $<0.0001$ & Chemotherapy & & & \\
\hline Urban $\geq 20,000$ adjacent to $\mathrm{MA}$ & 0.87 & $0.80-0.95$ & 0.001 & Yes (Reference) & 1.00 & & \\
\hline Urban $\geq 20,000$ not adjacent to MA & 0.76 & $0.65-0.87$ & 0.0001 & None & 1.34 & $1.21-1.48$ & $<0.0001$ \\
\hline Urban $<20,000$ adjacent to MA & 0.96 & $0.88-1.04$ & 0.27 & Hormone therapy & & & \\
\hline Urban $<20,000$ not adjacent to MA & 0.87 & $0.77-0.99$ & 0.0302 & Yes (Reference) & 1.00 & & \\
\hline Rural $<2,500$ adjacent to MA & 1.02 & $0.85-1.23$ & 0.81 & None & 2.28 & $2.21-2.36$ & $<0.0001$ \\
\hline
\end{tabular}

Great circle distance

\begin{tabular}{lccc}
$<50$ miles (Reference) & 1.00 & & \\
$\geq 50$ miles & 1.36 & $1.26-1.47$ & $<0.0001$ \\
Primary payor & & & \\
$\quad \begin{array}{l}\text { Private insurance (Reference) } \\
\text { Uninsured }\end{array}$ & 1.00 & & \\
Medicaid & 1.34 & $0.99-1.82$ & 0.06 \\
Medicare & 1.25 & $1.06-1.49$ & 0.0088 \\
Other government & 1.03 & $0.98-1.08$ & 0.23 \\
& 1.03 & $0.79-1.36$ & 0.81 \\
\hline
\end{tabular}

significant, there were no significant differences in axillary recurrence, breast cancer specific survival or distant metastasis. The 5 -year OS was $93.9 \%$ in both groups $(p=0.34)$ and most of the deaths were attributed to non-breast cancer related deaths. These authors arrived at the same conclusion as those investigators of the CALGB 9343 trial.

MA: Metropolitan area. *Percentage of adults (age $\geq 25$ years) who did not graduate from high school in the area based on the 2012 American Community Survey data. Odds ratios and $95 \%$ confidence interval values were rounded to the nearest 10th.

Although phase III clinical trials are considered "goldstandard" for which clinicians depend on to practice evidencebased medicine, the degree to which they are adopted by the medical community might not be as dramatic as one might anticipate. The impact of CALGB 9343 on practice patterns was evaluated by three recent publications (2-4). Soulos et al . (2) evaluated 12,925 patients from the Surveillance, Epidemiology, and End Results (SEER)-Medicare data set and found that the overall absolute reduction of RT usage following CALGB publication was approximately 4.3\%, 
which is slightly greater than our finding of $3.37 \%$. Palta $e t$ al. (3) decided to evaluate the entire SEER database and found an absolute reduction of $6.9 \%$ in RT usage following CALGB publication. Finally, McCormick et al. (4) examined 1,292 patient data from 13 National Comprehensive Cancer Network (NCCN) centers and found that the absolute reduction in RT usage was $9 \%$ following CALGB publication.

One of the limitations of using the SEER-Medicare or SEER data sets is the short interval between publication of CALGB and last follow-up date [data were based on those 3 years after CALGB publication for Soulos et al. (2) and 5 years for Palta et al. (3)] The limitations with McCormick et al.'s analysis (4) include a limited number of centers being evaluated. Of the 23 National Cancer Institute-designated Cancer Centers, only 13 institutions were represented. Our analysis using $\mathrm{NCDB}$, on the other hand, is clinical rather than administrative in nature. NCDB represents $70 \%$ of newly diagnosed cancer cases in the US whereas the SEER database represents only $28 \%$ of the US population. Regardless of this, the absolute reduction of RT following CALGB publication was exactly the same between our study and the SEER-Medicare study (4\%). This likely reflects that $\mathrm{CoC}$ hospitals in $\mathrm{CoC}$ regions use the same registrar and registry that submit data to both SEER and $\mathrm{CoC}$.

One of the advantages with the NCDB is that it has information on other factors such as comorbidity index, population density, facility type, and facility location. These last few variables allow us to understand the variation of practice patterns among the different regions as well as the different facilities throughout the US. Our time interval between publication of CALGB and last follow-up was also longer ( 8 years) compared to the other studies. Finally, our study comprises of over 120,000 patients, making our analysis not only a robust one, but also the largest one that also reflects the general practice of the US population.

A major limitation of our study is the lack of having complete data on adjuvant treatment, especially for adjuvant anti-hormone therapy. NCDB fail to capture the use of antihormone therapy in upwards $30 \%$ of cases and radiation therapy in upwards $20 \%$ of cases. Such limitation may partly explain the apparent omission of radiation and endocrine therapy in the same patients.

Similar to findings of others, we found that RT usage was significantly low among those at the extreme of ages and those with high comorbidity index (2-4). However, even among those at the extreme of ages, RT usage remains substantial; almost $33 \%$ of octogenarians ages $\geq 85$ years received RT following CALGB publication. Palta et al. (3) and McCormick et al. (4) also reported similar observation in their studies, mainly that between $30 \%-44 \%$ of patients ages $\geq 80$ years received RT.

Those patients with smaller tumors were more likely to not receive RT compared to larger size tumors. This might be
Table III. Type of radiation therapy administered

\begin{tabular}{lcc}
\hline $\begin{array}{l}\text { Regional treatment } \\
\text { modality, } \mathrm{N}=81,703\end{array}$ & $\begin{array}{c}\text { Pre-CALBG, } \\
\%\end{array}$ & $\begin{array}{c}\text { Post-CALBG, } \\
\%\end{array}$ \\
\hline External beam & 82.9 & 65.1 \\
IMRT & 4.7 & 9.3 \\
Conformal or 3-D & 5.2 & 10.8 \\
Brachytherapy & 6.3 & 13.8 \\
Other type & 0.9 & 0.9 \\
\hline
\end{tabular}

CALGB: Cancer and Leukemia Group B; pre-CALGB: up to 2004; post-CALGB: 2005-2012; IMRT: intensity-modulated radiation therapy; 3-D: 3-dimensional.

expected since the argument could be made that smaller tumors may carry a less aggressive course than larger tumors. What is unexpected is that patients with residual tumor were less likely to receive RT compared to those with no residual tumor. The reason behind this is not clear to us. Perhaps residual tumor is a surrogate marker of older age patients and/or those with higher comorbidity index. In other words, older patients or those with high comorbidity index were more likely to have residual tumor following a lumpectomy, and because of their presumed fragile status, they were less likely to be offered RT. Our analysis (not shown) did find a correlation between advanced age and evidence of residual tumor. Similarly, there was a direct correlation between residual tumor and high comorbidity index. What this implies is that surgeons should make an attempt to achieve negative margins for those in the extreme of ages and/or high comorbidity index because they are less likely to receive RT.

The literature reported that substantial racial disparities exist in the use of RT after breast-conserving surgery for invasive breast cancer. Compared to White women, African-American women were reportedly less likely to receive adjuvant RT following breast-conserving surgery. In our data, we also noted that elderly African-American women were less likely to receive adjuvant $\mathrm{RT}$ following breast-conserving surgery. It is difficult to discern whether this observation was related to the higher adherence rate to non-use of RT for African-American women or a reflection of racial/ethnic disparity.

Academic research programs were more likely to omit RT following CALGB publication compared to community cancer programs and comprehensive community cancer centers. Such institutional differences were also observed by McCormick $e t$ al. (4); even among NCCN institutions, RT omission ranged from $7-51 \%$. Our data are informative in that they allow centers across the different regions of the US to evaluate their practice patterns and determine whether it is necessary to make changes in order to adhere to evidence-based medicine.

It has been proposed that resistance to omitting RT may be due to financial incentives associated with provision of adjuvant RT (2). Our data appear to support this position. 
Table IV. Summary of literature evaluating impact of Cancer and Leukemia Group B (CALGB) 9343 trial.

\begin{tabular}{|c|c|c|c|c|}
\hline Author, year (Ref) & Database & $\mathrm{N}$ & $\begin{array}{l}\text { Absolute reduction of } \mathrm{RT} \\
\text { post CALGB publication }\end{array}$ & $\begin{array}{l}\text { Factors associated } \\
\text { with RT omission }\end{array}$ \\
\hline Soulos et al., 2012 (2) & SEER-Medicare & 12,295 & $4 \%$ & $\begin{array}{c}\text { Increasing age } \\
\text { High comorbidity index } \\
\text { Unmarried or unknown marital status }\end{array}$ \\
\hline McCormick et al., 2014 (4) & $\mathrm{NCCN}$ & 1,292 & $9 \%$ & $\begin{array}{c}\text { No axillary surgery } \\
\text { Small tumor } \\
\text { High comorbidity index } \\
\text { Varied among NCCN institution }\end{array}$ \\
\hline Palta et al., 2015 (3) & SEER & 40,583 & $7 \%$ & $\begin{array}{c}\text { Increasing age } \\
\text { Small tumor } \\
\text { High tumor grade } \\
\text { Right breast }\end{array}$ \\
\hline Current study & NCDB & 120,308 & $4.04 . \%$ & $\begin{array}{c}\text { Increasing age } \\
\text { African-American } \\
\text { High comorbidity index } \\
\text { Small tumor } \\
\text { Low tumor grade } \\
\text { Residual tumor } \\
\text { No chemotherapy } \\
\text { No anti-hormonal therapy } \\
\text { Medicaid } \\
\text { Low Income } \\
\text { Far distance } \\
\text { Facility type* } \\
\text { Facility location* } \\
\text { Population density* }\end{array}$ \\
\hline
\end{tabular}

SEER: Surveillance, Epidemiology, and End Results; NCCN: National Comprehensive Cancer Network; NCDB: National Cancer Data Base. *Varied (see text).

Medicaid patients were more likely not to receive RT compared to those with private insurance.

Another plausible explanation for the low adoption is provider biases and differences in interpretation of clinical trials (4). It is thought that the medical community is more likely to respond to trials that add new treatment rather than withholding old ones $(2,4,14)$. Finally, at the time of the initial CALGB results, clinicians might have felt that it was too early to embrace the new guideline given that the 5-year median follow-up time of the study was considered insufficient (2). Perhaps given longer follow-up results for CALGB 9343 (12) and the recent PRIME II validation study (13), we might see a more dramatic adoption of omitting RT use over time. However, whether or not this will actually happen will not be known for years to come.

One of the arguments in favor of use of RT is that not all 70 -year-olds are the same. It has been estimated that the life expectancy of a 70-year-old woman is more than 16 years (15). Although survival may not be affected by RT, recurrence can impose a psychological burden for which its importance should not be minimized. An alternate RT modality (i.e. brachytherapy) that imposes fewer burdens on patient time and schedule may partly explain the low adoption rate. In our study, we found that while $83 \%$ of patients had external beam radiation in the pre-CALGB era, this decreased to $65 \%$ in the post-CALGB era (Table III). Brachytherapy usage increased from $6.3 \%$ in the pre-CALGB era to $13.8 \%$ in the postCALGB era. The shorter time that it takes for a patient to complete RT with brachytherapy (i.e. 5-day course with brachytherapy versus 5 days per week for up to 6 weeks with external beam radiotherapy) may be a compelling argument in favor of continued utilization of RT, especially for those who are medically fit.

Finally, with our large data analysis showing poor rates of acceptance of RT omission in low-risk elderly patients, along with growing level I evidence supporting the safety of omitting RT in this patient population, it may be time to acknowledge and integrate omission of RT as part of the CoC benchmark for low-risk elderly patients undergoing breast-conserving therapy.

\section{Conclusion}

In summary, we report an analysis of the largest series of patients $(120,308)$ who fit CALGB 9343 criteria and evaluated the practice patterns of omitting adjuvant RT since 
the publication of CALGB 9343. We found that although there were statistically significant differences in the decrease in RT use between the groups, the absolute differences were incredibly small, and likely not clinically significant. Thus, there was a minimal impact ( $\sim \%$ reduction in RT usage) on practice patterns following CALGB publication. Specific socioeconomic factors such as education level had no bearing on the rate of RT utilization. Omission of RT was more likely among elderly patients with small, $\mathrm{ER}^{+}$breast cancer with the following criteria: extreme age group, high comorbidity index, therapy under an academic research program, higher great circle distance to treatment center, small tumor size, well-differentiated histology, residual tumor, and lack of receipt of chemotherapy and antihormonal therapy. Omission of RT also varies widely based on geographic location.

\section{Acknowledgements}

The data used in the study were derived from a de-identified NCDB file. The American College of Surgeons and the Commission on Cancer have not verified and are not responsible for the analytic or statistical methodology employed, or the conclusions drawn from these data by the investigator.

This work was supported by the Charles D. Knight, Sr. Endowed Professor of Surgery.

\section{References}

1 Hughes KS, Schnaper LA, Berry D, Cirrincione C, McCormick B, Shank B, Wheeler J, Champion LA, Smith TJ, Smith BL, Shapiro C, Muss HB, Winer E, Hudis C, Wood W, Sugarbaker D, Henderson C, Norton L, for the Cancer and Leukemia Group B, Radiation Therapy Oncology Group, and Eastern Cooperative Oncology Group: Lumpectomy plus tamoxifen with or without irradiation in women 70 years of age or older with early breast cancer. N Engl J Med 351(10): 971-977, 2004.

2 Soulos PR, Yu JB, Roberts KB, Raldow AC, Herrin J, Long JB and Gross CP: Assessing the impact of a cooperative group trial on breast cancer care in the medicare population. J Clin Oncol 30(14): 1601-1607, 2012.

3 Palta M, Palta P, Bhavsar NA, Horton JK and Blitzblau RC: The use of adjuvant radiotherapy in elderly patients with early-stage breast cancer: changes in practice patterns after publication of Cancer and Leukemia Group B 9343. Cancer 121(2): 188-193, 2015.

4 McCormick B, Ottesen RA, Hughes ME, Javid SH, Khan SA, Mortimer J, Niland JC, Weeks JC and Edge SB: Impact of guideline changes on use or omission of radiation in the elderly with early breast cancer: practice patterns at National Comprehensive Cancer Network institutions. J Am Coll Surg 219(4): 796-802, 2014.
5 Categories of Accreditation. http://www.facs.org/cancer/coc/ categories.html. Accessed November 9, 2014.

6 Charlson ME, Pompei P, Ales KL and MacKenzie CR: A new method of classifying prognostic comorbidity in longitudinal studies: development and validation. J Chronic Dis 40(5): 373383, 1987.

7 Deyo RA, Cherkin DC and Ciol MA: Adapting a clinical comorbidity index for use with ICD-9-CM administrative databases. J Clin Epidemiol 45(6): 613-619, 1992.

8 Fisher B, Bauer M, Margolese R, Poisson R, Pilch Y, Redmond C, Fisher E, Womark N, Deutsch M, Montague E, Saffer E, Wickerham L, Lerner H, Glass A, Shibata H, Deckers P, Ketcham A, Oishi R and Russel I: Five-year results of a randomized clinical trial comparing total mastectomy and segmental mastectomy with or without radiation in the treatment of breast cancer. N Engl J Med 312(11): 665-673, 1985.

9 Early Breast Trialists Collaborative Group (EBCTCG), Darby S, McGale P, Correa C, Taylor C, Arriagada R, Clarke M, Cutter D, Davies C, Ewertz M, Godwin J, Gray R, Pierce L, Whelan T, Wang $\mathrm{Y}$ and Peto R: Effect of radiotherapy after breastconserving surgery on 10-year recurrence and 15-year breast cancer death: meta-analysis of individual patient data for 10,801 women in 17 randomised trials. Lancet 378(9804): 1707-1716, 2011.

10 Fisher B, Jeong JH, Anderson S, Bryant J, Fisher ER and Wolmark N: Twenty-five-year follow-up of a randomized trial comparing radical mastectomy, total mastectomy, and total mastectomy followed by irradiation. N Engl J Med 347(8): 567575, 2002.

11 Carlson RW and Edge SB: Update: NCCN breast cancer clinical practice guidelines. J Natl Compr Canc Netw 2(Suppl 3): S-1-4, 2004.

12 Hughes KS, Schnaper LA, Bellon JR, Cirrincione CT, Berry DA, McCormick B, Muss HB, Smith BL, Hudis CA, Winer EP and Wood WC: Lumpectomy plus tamoxifen with or without irradiation in women age 70 years or older with early breast cancer: long-term follow-up of CALGB 9343. J Clin Oncol 31(19): 2382-2387, 2013.

13 Kunkler IH, Williams LJ, Jack WJ, Cameron DA, Dixon JM, PRIME II investigators: Breast-conserving surgery with or without irradiation in women aged 65 years or older with early breast cancer (PRIME II): a randomised controlled trial. Lancet Oncol 16(3): 266-273, 2015.

14 Giordano SH: Radiotherapy in older women with low-risk breast cancer: why did practice not change? J Clin Oncol 30(14): 15771578, 2012.

15 Find the Data: Compare life span expectancy and mortality rates. In: http://life-span.findthedata.org.
Received August 11, 2017

Revised September 8, 2017

Accepted September 13, 2017 